Kansas State University Libraries

New Prairie Press

\title{
EVALUATING PEN-DAY INTERACTIONS IN BODY TEMPERATURE BILOGISTIC MIXED MODEL FOR HANDLING OF FEEDLOT HEIFERS DURING HEAT STRESS
}

F. Yang

A. M. Parkhurst

T. M. Brown-Brandl

R. A. Eigenberg

J. A. Nienaber

See next page for additional authors

Follow this and additional works at: https://newprairiepress.org/agstatconference

Part of the Agriculture Commons, and the Applied Statistics Commons (c) (1) (9)

This work is licensed under a Creative Commons Attribution-Noncommercial-No Derivative Works 4.0 License.

\section{Recommended Citation}

Yang, F.; Parkhurst, A. M.; Brown-Brandl, T. M.; Eigenberg, R. A.; and Nienaber, J. A. (2010). "EVALUATING PEN-DAY INTERACTIONS IN BODY TEMPERATURE BILOGISTIC MIXED MODEL FOR HANDLING OF FEEDLOT HEIFERS DURING HEAT STRESS," Conference on Applied Statistics in Agriculture. https://doi.org/10.4148/2475-7772.1059

This is brought to you for free and open access by the Conferences at New Prairie Press. It has been accepted for inclusion in Conference on Applied Statistics in Agriculture by an authorized administrator of New Prairie Press. For more information, please contact cads@k-state.edu. 


\section{Author Information}

F. Yang, A. M. Parkhurst, T. M. Brown-Brandl, R. A. Eigenberg, and J. A. Nienaber 


\title{
EVALUATING PEN-DAY INTERACTIONS IN BODY TEMPERATURE BILOGISTIC MIXED MODEL FOR HANDLING OF FEEDLOT HEIFERS DURING HEAT STRESS
}

\author{
F. Yang ${ }^{1}$, A. M. Parkhurst ${ }^{1}$, T.M. Brown-Brandl ${ }^{2}$, R.A. Eigenberg ${ }^{2}$, and J.A. \\ Nienaber $^{2}$ \\ 1. Department of Statistics, University of Nebraska at Lincoln \\ 2. USDA-ARS U.S. Meat Animal Research Center
}

\begin{abstract}
Daily activities consume the energy of heifers, subsequently causing an elevation of body temperature, depending on the ambient conditions. A better understanding of the dynamics of body temperature (Tb) would be helpful when deciding how to process and handle heifers. It would also lead to specific recommendations on moving heifers under different ambient conditions, especially during the summer. In this study, a bilogistic mixed model is used to describe the dynamics of Tb during the moving event. Data was taken from heifers in pens located at different distances from the heifer work station on four separate summer days under hot conditions. This bilogistic model has seven biological parameters: initial body temperature, heat challenge rate constant, upper asymptote body temperature, challenge inflection point, baseline body temperature for recovery, recovery rate constant, and recovery inflection point. Pen and day were used as treatment factors in the model. Significant interactions between the factors were found for several parameters, indicating distance moved during the handling event influences the way an animal responds to a thermal challenge. The objectives of this study are to fit a bilogistic mixed model for Tb with the above seven parameters, and to examine fixed and random effects. The main focus is to estimate and interpret the interactions between pens and days for the significant parameters to aid in management decisions involving when to work cattle.
\end{abstract}

Key words: Working cattle, Moving distances, Thermo-regulatory response, Ambient temperature-distance interactions, Dynamics of body temperature.

\section{INTRODUCTION}

The cattle industry is one of the most important and profitable industries in the U.S. Profit from cattle industry is related to an animal's condition and environment. Heat stress can be a serious problem for cattle in a hot working environment. It is the major cause of lost profits in hostile "production-limiting" regions. Animals suffer heat stress when temperature exceeds some threshold. When heat stress happens, it will slow cattle growth and reduce cattle feeding and production, and will even result in death of susceptible cattle. Eventually, it will bring large economic losses. Therefore, understanding when an animal is experiencing heat stress can provide a basis for initiation of management practices to improve an animal's well being. (Davis et al. 2003) 
Body temperature ( $\mathrm{Tb}$ ) is a good way to measure an animal's thermo-regulatory response to the environment. It found that heat stress in working cattle can be measured as the temperature differential, i.e., the difference between the upper asymptote and initial body temperature. Other parameters, such as challenge rate constant, recovery rate constant, challenge inflection point, recovery inflection point and recovery baseline body temperature, are also associated with heat production.

Parkhurst and Mader (2000) showed fitting parameters of a nonlinear bilogistic model to $\mathrm{Tb}$ provides insight into the process of handling and moving animals during thermal challenge conditions of summer. Therefore, in this study, a nonlinear mixed bilogistic model with seven parameters is used to describe heifer's body temperature during moving event in a hot environment. A cross-over experiment design using pen and day as factors is analyzed. The objectives of this study are: 1) to derive an improved bilogistic model which can indicate the dynamics of $\mathrm{Tb}$ during thermal challenge and recovery, 2) to fit an appropriate mixed bilogistic model with pen (or distance) and day treatment factors, 3) examine the fixed and random effects in the mixed model and examine the pen-day interactions.

\section{MATERIALS and METHOD}

\subsection{Materials and Experimental Design}

The data consisted of measurements taken from thirty-two 4-breed composite heifers (1/4 Pinzgauer, 1/4 Red Poll, $1 / 4$ Hereford, and 1/4 Angus; initially weighing 420 \pm 43.9 kg). Heifers were randomly selected and assigned to one of four pens on the basis of weight and health history.

Over the 10 week trial period, experiment was conducted on two separate dates (6/26/2007, 8/1/2007) which had different average ambient temperature during a working event where the animals were processed in a squeeze chute. Pens were equally spaced approximately 160 - $200 \mathrm{~m}$ from a working facility. Pen 2 was located the furthest away, while pen 8 was the closest to the facility. In each working event, heifers were moved from their pen to the working facility during a hot environmental challenging. Body temperature $(\mathrm{Tb})$ were recorded from 60 minutes prior to leaving the pen and then for the next 4 hours and on a one minute basis. In this study, Tb data for 90 minutes after heifers were moved were analyzed. Table 1. A 5\% significance level was chosen for all analyses in this study

Table 1. 32 heifers randomly assigned to 4 pens on 2days.

\begin{tabular}{|c|c|c|c|}
\hline \multicolumn{2}{|c|}{ Distance } & 6/26/2007 Cool & 8/1/2007Hot \\
\hline Pen 2 & \multirow{4}{*}{ Furthest $200 \mathrm{~m}$} & Heifers 1-8 & Heifers 1-8 \\
\hline Pen 4 & & Heifers 16-24 & Heifers 16-24 \\
\hline Pen 6 & & Heifers 25-32 & Heifers 25-32 \\
\hline Pen 8 & & Heifers 33-40 & Heifers 33-40 \\
\hline
\end{tabular}




\subsection{Model}

Parkhurst and Mader (2000) used a logistic relationship to describe the dynamics of Tb during thermal challenge or recovery. Parkhurst and Mader also suggested that a nonlinear mixed bilogistic model could be used to describe Tb during the handling process. To provide a better prediction and interpretation, an improved bilogistic model needs to be derived and treatment effects need to be analyzed.

\section{2.a. Build An Additive Bilogistic Model}

When an animal experiences a thermal challenge, the relative rate of heat accumulation is not constant but is proportional to the amount of heat remaining from the upper asymptote Tb; i.e. a logistic relationship, (Parkhurst and Mader 2000). Similarly, when an animal eliminates heat during thermal recovery, the relative recovery rate is proportional to the amount of heat above the baseline. Assuming that challenge and recovery are independent, an additive bilogistic model is obtained by combining both logistic relationships. The two processes can be thought of as describing thermal challenge and thermal recovery.

\section{2.a.1. Individual Logistic functions}

During the thermal challenge period, let $Y=T b-\beta_{1}$, where $T b\left({ }^{\circ} \mathrm{C}\right)$ is the body temperature at time $t(\mathrm{~min})$, which starts at $0 \mathrm{~min} ; \alpha$ is the upper asymptote $T b\left({ }^{\circ} \mathrm{C}\right)$ indicating the highest $\mathrm{Tb}$ that heifer was expected to achieve in the absence of a recovery process; $\beta_{1}$ $\left({ }^{\circ} \mathrm{C}\right)$ is the lower asymptote $T b$ during thermal challenge, which is the lowest body temperature in the 60 min's observation period before heifer leaves the pen. Then,

$$
\frac{\partial Y_{\text {challenge }}}{Y_{\text {challenge }} \partial t}=k_{1}\left(\alpha-\beta_{1}-Y_{\text {challenge }}\right) \text {, with } \frac{\partial Y_{\text {challenge }}}{Y_{\text {challenge }} \partial t}>0 ; \alpha-\beta_{1}>Y_{\text {challenge }} ; k_{1}>0 \text {. }
$$

Suppose $T b(0)=T b_{\text {init }}$, (i.e., $T b$ at $t=0$ is $T b_{\text {init, }}$ and $T b_{\text {init }} \neq \beta_{1}$ ), then the initial condition is $Y_{\text {challenge }}(0)=T b(0)-\beta_{1}=T b_{\text {init }}-\beta_{1}$.

Hence,

$$
Y_{\text {challenge }}=\frac{\alpha-\beta_{1}}{1+e^{-k_{1} t}\left(\frac{\alpha-T b_{\text {init }}}{T b_{\text {init }}-\beta_{1}}\right)} \Leftrightarrow T b=\frac{\alpha-\beta_{1}}{1+e^{-k_{1} t}\left(\frac{\alpha-T b_{\text {init }}}{T b_{\text {init }}-\beta_{1}}\right)}+\beta_{1}
$$

Let $\frac{\alpha-T b_{\text {init }}}{T b_{\text {init }}-\beta_{1}}=e^{k_{1} \tau_{1}}$, then $\beta_{1}=T b_{\text {init }}-\left(\alpha-T b_{\text {init }}\right) e^{-k_{1} \tau_{1}}$, where $\tau_{1}$ is the inflection (or change) point during the thermal challenge period.

Hence, when an animal experiences a thermal challenge, $T b$ can be written as

$$
T b_{\text {challenge }}=\frac{\left(\alpha-T b_{\text {init }}\right)\left(1-e^{-k_{1} t}\right)}{1+e^{-k_{1}\left(t-\tau_{1}\right)}}+T b_{\text {init }}
$$

Similarly for thermal recovery: 
$Y=T b-\beta_{2}$, where $T b$ is the body temperature at time $t ; \alpha$ is the upper asymptote Tb with the same meaning as in thermal challenge; $\beta_{2}\left({ }^{\circ} \mathrm{C}\right)$ is the lower asymptotic Tb during thermal recovery, which is the lowest body temperature that heifer would achieve in the absence of the thermal challenge. Then,

$$
\frac{\partial Y_{\text {recovery }}}{Y_{\text {recovery }} \partial t}=-k_{2}\left(\alpha-\beta_{2}-Y_{\text {recovery }}\right) \text {, with } \frac{\partial Y_{\text {recovery }}}{Y_{\text {recovery }} \partial t} \leq 0 ; \alpha-\beta_{2} \geq Y_{\text {recovery }} ; k_{2}>0
$$

Suppose the effect of handling lasts $90 \mathrm{~min}$, then $T b(90)=\delta$ when time is 90 (i.e. Tb at the ending point of the handling event is $\delta$ ), then $Y_{\text {recovery }}(90)=T b(90)-\beta_{2}=\delta-\beta_{2}$. Hence,

$$
Y_{\text {recovery }}=\frac{\alpha-\beta_{2}}{1+e^{k_{2}(t-90)}\left(\frac{\alpha-\delta}{\delta-\beta_{2}}\right)} \Leftrightarrow T b_{\text {recovery }}=\frac{\alpha-\beta_{2}}{1+e^{k_{2}(t-90)}\left(\frac{\alpha-\delta}{\delta-\beta_{2}}\right)}+\beta_{2}
$$

Let $e^{-k_{2} 90} \frac{\alpha-\delta}{\delta-\beta_{2}}=e^{-k_{2} \tau_{2}}$, then $\beta_{2}=\delta-(\alpha-\delta) e^{-k_{2}\left(90-\tau_{2}\right)}$, where $\tau_{2}$ is the inflection (or change) point for recovery.

Hence, when an animal experiences thermal recovery is

$$
T b_{\text {recovery }}=\frac{(\alpha-\delta)\left(1-e^{-k_{2}(90-t)}\right)}{1+e^{k_{2}\left(t-\tau_{2}\right)}}+\delta
$$

\section{2.a.2. Additive Bilogistic function}

Viewing the process as a whole, the differential equation can be considered to be the sum of the rate of change in each process. Thus, the overall rate of change is actually the rate of challenge plus the rate of recovery. It is a combination of two logistic processes. Therefore, it is named an additive bilogistic function.

$$
\begin{aligned}
& \frac{\partial T b}{\partial t}=\left.\frac{\partial T b}{\partial t}\right|_{\text {challenge }}+\left.\frac{\partial T b}{\partial t}\right|_{\text {recovery }}=\frac{\partial\left(Y_{\text {challenge }}+\beta_{1}\right)}{\partial t}+\frac{\partial\left(Y_{\text {recovery }}+\beta_{2}\right)}{\partial t}=\frac{\partial Y_{\text {challenge }}}{\partial t}+\frac{\partial Y_{\text {recovery }}}{\partial t} \\
& \frac{\partial T b}{\partial t}=k_{1} Y_{\text {challenge }}\left(\alpha-\beta_{1}-Y_{\text {challenge }}\right)-k_{2} Y_{\text {recovery }}\left(\alpha-\beta_{2}-Y_{\text {recovery }}\right)
\end{aligned}
$$

with initial condition $T b(0)=T b_{\text {init }}$ and ending condition $T b(90)=\delta$. Then,

$$
T b(t)=\alpha-\left(\alpha-T b_{\text {init }}\right)\left(\frac{e^{-k_{1} \tau_{1}}+1}{e^{k_{1}\left(t-\tau_{1}\right)}+1}\right)-(\alpha-\delta)\left(\frac{e^{k_{2}\left(\tau_{2}-90\right)}+1}{e^{-k_{2}\left(t-\tau_{2}\right)}+1}\right)
$$

where $\alpha, \delta, T b_{\text {init }}, k_{1}, k_{2}, \tau_{1}$, and $\tau_{2}$ are all positive.

$\alpha$ is the upper asymptotic body temperature (not the maximum $\mathrm{Tb}$ ),

$T b_{\text {init }}$ is animal's initial body temperature

$\delta$ is animal's baseline body temperature for recovery.

$k_{1}$ and $k_{2}$ are rate constants for challenge and recovery periods, respectively.

$\tau_{1}$ and $\tau_{2}$ are inflection points for challenge and recovery periods, respectively. 
Fig1. Plots of simulated logistic models and simulated additive bilogistic model, for Tb over 90 min using $\alpha=40.015$ (such that $T b_{\max }=40$ ), $T b_{\text {init }}=38, \delta=39, k_{1}=0.12$, $k_{2}=0.12, \tau_{1}=20, \tau_{2}=70$.
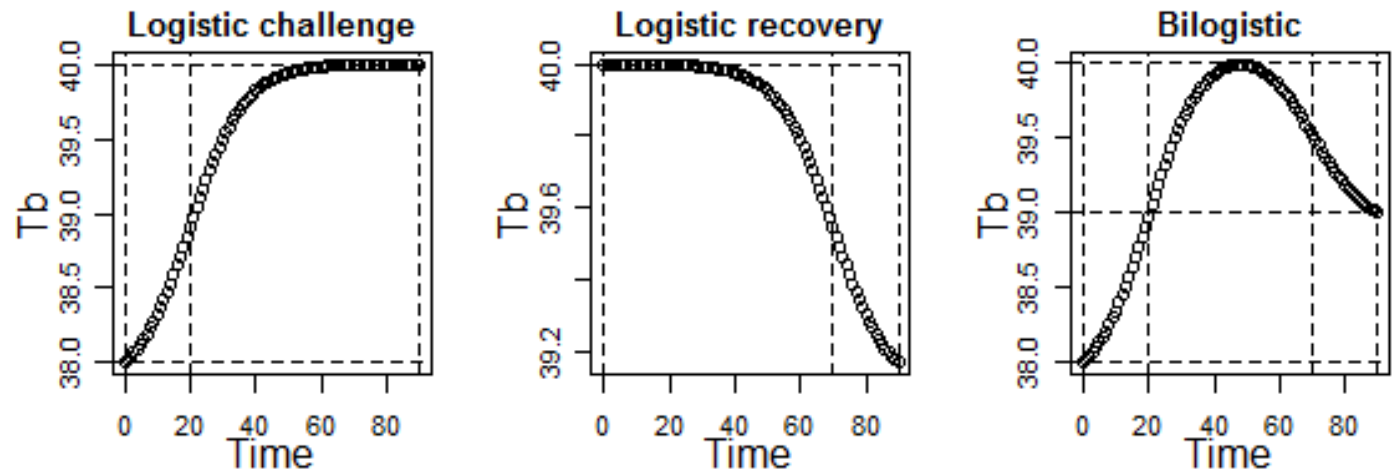

\section{2.b. Mixed Additive Bilogistic Model with Treatment Effects}

The additive bilogistic model provides a way to describe Tb during the handling event. A nonlinear mixed model with fixed effects and random heifer effects needs to be considered. In this study, 2 factors (day and pen) and 7 random effects (one for each of the seven parameters) were incorporated in additive bilogistic model.

\section{2.b.1. Random Heifer Effects}

Two random structures for grouping animals, individual cluster (crossed) and hierarchical cluster (nested) were analyzed in this study. For one model the grouping structure factors are said to be crossed (individual cluster) if every level of one factor occurs with every level of another factor, and vice verse. The structure is considered to be nested within another factor (Hierarchical cluster) if every level of this factor occurs with only one level of the other factor.

\section{2.b.1.i. Individual Clusters or Crossed Random Structure}

In this structure, each factor combination is considered a separate group. Data were grouped by a factor "heiferDay" which was a combination of heifer i.d. and day number. Thus, the random effect in this structure was clustered by heiferDay.

The statistical model for individual clusters (crossed) structure is

$$
T b_{i}=\alpha-\left(\alpha-T b_{i n i t}\right)\left(\frac{e^{-k_{1} \tau_{1}}+1}{e^{k_{1}\left(t-\tau_{1}\right)}+1}\right)-(\alpha-\delta)\left(\frac{e^{k_{2}\left(\tau_{2}-90\right)}+1}{e^{-k_{2}\left(t-\tau_{2}\right)}+1}\right)+\varepsilon_{i}
$$




$$
\begin{aligned}
& {\left[\begin{array}{cc}
\alpha & =\mu_{\alpha}+b_{\alpha: \text { heiferDay }} \\
\delta & =\mu_{\delta}+b_{\delta: \text { heiferDay }} \\
k_{1} & =\mu_{k_{1}}+b_{k_{1} \text { hhifferDay }} \\
k_{2} & =\mu_{k_{2}}+b_{k_{2}: \text { heiffrDay }} \\
\tau_{1} & =\mu_{\tau_{1}}+b_{\tau_{1}} \text { heiferDay } \\
\tau_{2} & =\mu_{\tau_{2}}+b_{\tau_{2}: \text { heiferDay }} \\
T b_{\text {init }} & =\mu_{\text {init }}+b_{\text {init:heiferDay }}
\end{array}\right]}
\end{aligned}
$$

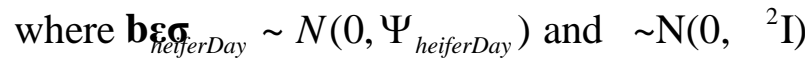

$$
\begin{aligned}
& \text { are independent from each other. } \\
& \Psi_{\text {heiferDay }}=\left[\begin{array}{cccc}
\psi_{\alpha: \text { heiferDay }} & 0 & \cdots & 0 \\
& \psi_{\text {S:heiferDay }} & & \vdots \\
& & \ddots & 0 \\
& & & \psi_{\text {init:heiferDay }}
\end{array}\right]
\end{aligned}
$$
random effects at heiferDay level. They present the deviations from the population mean associated with different heiferDay handling events.

\section{2.b.1.ii. Hierarchical Clusters or Nested random structure}

In a hierarchical clusters structure, the sub-samples are nested within the level-one sampling units. In this structure, data was grouped by "heifer/day". Thus the random effect has 2 levels: heifer and day within heifer. The nested random structure with grouping factor "heifer/day" helps to access the interaction between heifer and day.

The additive bilogistic model with both fixed and random effects for the nested structure is

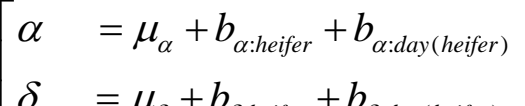

$$
\begin{aligned}
& k_{1}=\mu_{\kappa_{1}}+b_{k_{1} \text { :heifer }}+b_{k_{1} \text { :day(heifer) }} \\
& k_{2}=\mu_{\kappa_{2}}+b_{k_{2}: \text { heifer }}+b_{k_{2}: \text { day(heifer) }} \\
& \tau_{1}=\mu_{\tau_{1}}+b_{\tau_{1}: \text { heifer }}+b_{\tau_{1}: \text { day (heifer) }} \\
& \tau_{2}=\mu_{\tau_{2}}+b_{\tau_{2}: \text { heifer }}+b_{\tau_{2}: \text { day (heifer) }} \\
& T b_{\text {init }}=\mu_{\text {init }}+b_{\text {init:heifer }}+b_{\text {init:day(heifer) }} \\
& \text { where } \mathbf{b}_{\text {heifer }} \sim N\left(0, \Psi_{\text {heifer }}\right), \mathbf{b}_{\text {day (heifer })} \sim N\left(0, \Psi_{\text {day (heifer })}\right)
\end{aligned}
$$
and $\boldsymbol{\varepsilon} \boldsymbol{\sigma} \mathrm{N}\left(0,{ }^{2} \mathrm{I}\right)$ are independent from each other

$$
\begin{aligned}
& \Psi_{\text {heifer }}=\left[\begin{array}{ccc}
\psi_{\alpha: \text { heifer }} & \ldots & 0 \\
\vdots & \ddots & \vdots \\
0 & \ldots & \psi_{\text {init:heifer }}
\end{array}\right] \\
& \Psi_{\text {day_in_heifer }}=\left[\begin{array}{ccc}
\psi_{\alpha: \text { day (heifer })} & \ldots & 0 \\
\vdots & \ddots & \vdots \\
0 & \ldots & \psi_{\text {init:day(heifer) }}
\end{array}\right]
\end{aligned}
$$

In this model, $\boldsymbol{\mu}=\left(\mu_{\alpha}, \mu_{\delta}, \mu_{\kappa_{1}}, \mu_{\kappa_{2}}, \mu_{\tau_{1}}, \mu_{\tau_{2}}, \mu_{\text {init }}\right)$ ' are fixed effects of all seven parameters in the mixed model.

$\mathbf{b}_{\text {heifer }}=\left(b_{\alpha: \text { heifer }}, b_{\delta: \text { heifer }}, b_{\kappa_{1}: \text { hifer }}, b_{\kappa_{2}: \text { heifer }}, b_{\tau_{1}: \text { heifer }}, b_{\tau_{2}: \text { heifer }}, b_{\text {init:hifer }}\right)$ 'are the random effects of 
heifer. They represent the deviations from the population mean associated with heifer.

$\mathbf{b}_{\text {day (heifer) }}=\left(b_{\alpha: \text { day (heifer) }}, b_{\delta: \text { day (heifer) }}, b_{\left.k_{1}: \text { day (hiffer }\right)}, b_{\left.k_{2}: \text { day (hiffer }\right)}, b_{\tau_{1}: \text { day (heifer) }}, b_{\left.\tau_{2}: \text { day (heifer }\right)}, b_{\text {init:day(heifer) })}\right)^{\prime}$

are the random effect of day within heifer level. They represent the deviations from the population mean associated with each day for a heifer.

\section{2.b.2 Fixed Treatment Effects}

In this study, there were 2 treatment factors, day (2 levels) and pen (4 levels), which designate a $4 \mathrm{x} 2$ factorial treatment design. To determine if pen (distance) and Day (summer heat) have an effect on $\mathrm{Tb}$, fixed treatment effects need to be included in the model.

The statistic models for both random effect structures with treatment effect were given in (6) and (7).

$$
T b_{i}=\alpha-\left(\alpha-T b_{i n i t}\right)\left(\frac{e^{-k_{1} \tau_{1}}+1}{e^{k_{1}\left(t-\tau_{1}\right)}+1}\right)-(\alpha-\delta)\left(\frac{e^{k_{2}\left(\tau_{2}-90\right)}+1}{e^{-k_{2}\left(t-\tau_{2}\right)}+1}\right)+\varepsilon_{i}
$$

In an individual cluster (crossed) structure,

$$
\begin{aligned}
& {\left[\begin{array}{rl}
\alpha & =\sum_{i=1}^{2} \sum_{j=1}^{4} C_{i j} \beta_{\alpha, i j}+b_{\alpha: \text { heiferDay }} \\
\delta & =\sum_{i=1}^{2} \sum_{j=1}^{4} C_{i j} \beta_{\delta, i j}+b_{\delta: \text { heiferDay }} \\
k_{1} & =\sum_{i=1}^{2} \sum_{j=1}^{4} C_{i j} \beta_{k_{1}, i j}+b_{k_{1}: \text { heiferDay }} \\
k_{2} & =\sum_{i=1}^{2} \sum_{j=1}^{4} C_{i j} \beta_{k_{2}, i j}+b_{k_{2}: \text { heiferDay }} \\
\tau_{1} & =\sum_{i=1}^{2} \sum_{j=1}^{4} C_{i j} \beta_{\tau_{1}, i j}+b_{\tau_{1}: \text { heiferDay }} \\
\tau_{2} & =\sum_{i=1}^{2} \sum_{j=1}^{4} C_{i j} \beta_{\tau_{2}, i j}+b_{\tau_{2}: \text { heiffDay }} \\
T b_{\text {init }} & =\sum_{i=1}^{2} \sum_{j=1}^{4} C_{i j} \beta_{i n i t, i j}+b_{\text {init:heiferDay }}
\end{array}\right]} \\
& \Psi_{\text {heiferDay }}=\left[\begin{array}{cccc}
\psi_{\alpha: \text { heiferDay }} & 0 & \ldots & 0 \\
& \psi_{\text {s:heiferDay }} & & \vdots \\
& & \ddots & 0 \\
& & & \psi_{\text {init:heiferDay }}
\end{array}\right]
\end{aligned}
$$

$\mathrm{C}_{\mathrm{ij}}$ 's are dummy variables defined to incorporate the treatment effects, i.e., if the first day and pen 2 represents, $C_{11}=1$ and all other $C_{i j}{ }^{c} s=0$.

$\beta_{\text {. } i j}$ represents the mean of one parameter on the $i^{\text {th }}$ day and in $j^{\text {th }}$ pen.

$b$.heiferDay 's have the same meaning as in section 2.2.b.1.i. They are the random effects at heiferDay level. They present the deviations from the population mean associated with different heiferDay handling events. 
In a Hierarchical clusters (nested) structure,

$$
\begin{aligned}
& {\left[\alpha=\sum_{i=1}^{2} \sum_{j=1}^{4} C_{i j} \beta_{\alpha, i j}+b_{\alpha: \text { heifer }}+b_{\alpha: \text { day (heifer) }}\right] \text { where } \mathbf{b}_{\text {heifer }} \sim N\left(0, \Psi_{\text {heifer }}\right) \text {, }} \\
& \delta=\sum^{2} \sum^{4} C_{j, j}+b_{\delta \text { day (hifer) }} \sim N\left(0, \Psi_{\text {day(heifer })}\right)
\end{aligned}
$$

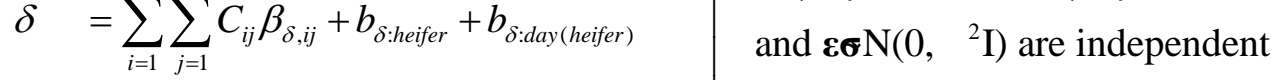

$$
\begin{aligned}
& k_{1}=\sum_{i=1}^{2} \sum_{j=1}^{4} C_{i j} \beta_{k_{1}, i j}+b_{k_{1}: \text { heifer }}+b_{\left.k_{1}: \text { day (heifer }\right)} \\
& k_{2}=\sum_{i=1}^{2} \sum_{j=1}^{4} C_{i j} \beta_{k_{2}, i j}+b_{k_{2}: \text { heifer }}+b_{\left.k_{2}: \text { day (heifer }\right)} \\
& \tau_{1} \quad=\sum_{i=1}^{2} \sum_{j=1}^{4} C_{i j} \beta_{\tau_{1}, i j}+b_{\tau_{1}: \text { hiffer }}+b_{\left.\tau_{1}: \text { day (heifer }\right)} \\
& \tau_{2} \quad=\sum_{i=1}^{2} \sum_{j=1}^{4} C_{i j} \beta_{\tau_{2}, i j}+b_{\tau_{2}: \text { heifer }}+b_{\left.\tau_{2}: \text { day (heifer }\right)} \\
& T b_{\text {init }}=\sum_{i=1}^{2} \sum_{j=1}^{4} C_{i j} \beta_{\text {init }, i j}+b_{\text {init:heifer }}+b_{\text {init:day (heifer })} \\
& \Psi_{\text {heifer }}=\left[\begin{array}{ccc}
\psi_{\alpha: \text { hiffer }} & \ldots & 0 \\
\vdots & \ddots & \vdots \\
0 & \ldots & \psi_{\text {init:heifer }}
\end{array}\right] \\
& \Psi_{\text {day (heifer) }}=\left[\begin{array}{ccc}
\psi_{\alpha: \text { day (heifer) }} & \ldots & 0 \\
\vdots & \ddots & \vdots \\
0 & \ldots & \psi_{\text {init:day (heifer) }}
\end{array}\right]
\end{aligned}
$$

$\mathrm{C}_{\mathrm{ij}}$ 's are dummy variables defined to incorporate the treatment effects, i.e., if the first day and pen 2 represents, $\mathrm{C}_{11}=1$ and all other $\mathrm{C}_{\mathrm{ij}}{ }^{\mathrm{c}} \mathrm{s}=0$.

$\beta$. ij represents the mean of one parameter on the $i^{\text {th }}$ day and in $j^{\text {th }}$ pen.

$b_{\text {.heifer }}$ and $b_{\text {.day(heifer) }}$ have the same meaning as in section 2.2.b.1.ii.

$b_{\text {.heifer }}$ 's are the random effects of heifer of one parameter. They represent the deviations from the population mean associated with heifer.

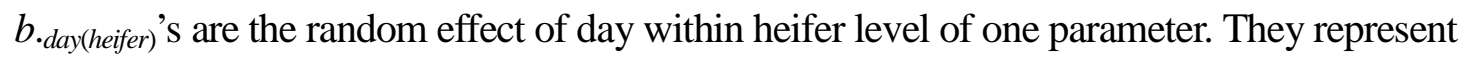
the deviations from the population mean associated with each day for a heifer.

\section{2.b.3. Comparison of mixed bilogistic models}

After fixed treatment effects and random heifer effects were specified, the four models were compared using log-likelihood, Information Criteria (AIC and BIC), and fitted plots. The model with higher log-likelihood, smaller AIC and BIC, and no unusual residuals would be preferred. The likelihood ration test (LRT) was also constructed between two models to test if one was significantly better than the other.

The mixed bilogistic model had seven parameters. Due to the difficulty in obtaining convergence for the optimization algorithm used in NLME (Pinheiro and Bates, 2000), a diagonal random structure was assumed for the random-effects covariance matrices. To identify which parameters, if any, require random effects, the mixed models in both cases were fit for several sets of diagonal random effects structures and checked for model equivalency. Each time, one random effect was removed, the reduced model was compared to the full model with all diagonal random effects using the LRT. If the LRT is not significant, then the removal of random-effect was justified. 


\section{RESULTS and DISCUSSION}

\subsection{Mixed Additive bilogistic model}

A mixed model with diagonal random-effects structure was used to examine heifer effects associated with each treatment factor. To find the best mixed model, comparisons were made between two groupings of random heifer effects, individual (crossed) and hierarchical (nested) clusters, both with and without treatment effects. The goodness-of-fit statistics for comparing the four models are summarized in Table 3.

For the models without treatment effects, the individual cluster (crossed) random-effects structure (Model 1) had smaller AIC, BIC, but larger log-likelihood compared to the (nested) clusters structure (Model 3) and the p-value for LRT was less than $5 \%$. (not shown). Hence, a mixed additive bilogistic model with crossed random effect structure was better than a nested random effect structure when treatment was not included.

For each random-effects structure comparisons were made between models with and without treatments effect. After treatment effects were included in the mixed model, AIC and BIC were smaller and log-likelihood statistics were larger when compared to models without treatment effect. For the both random-effects structures, the p-value for LRT was less than 5\%. (Model 1 vs 3 and Model 2 vs 4, not shown). Hence, the treatment effect is needed in the model regardless of the random-effects structure.

Comparing model 4 (with treatment effects and nested random structure) with the other three models showed model 4 had the smallest AIC and BIC, largest log-likelihood and a better fit on the prediction plot, Fig 2. The p-values for three LRTs (Model No. 1 vs 4, 2 vs 4 , and 3 vs 4 ) were all less than $5 \%$ which indicate the nested model with treatment effect was significantly better than the other three models. Figure 2 showed that the predicted data provided a good fit to the observed data at each day within heifer level, though some were underestimated. The residual analysis (not shown) supported the assumptions that residuals from model 4 at each day within heifer level were normal and randomly distributed about the zero line without any outliers. Therefore, the preferable model is the mixed bilogistic model with nested cluster random effects.

Table 2. Statistics from mixed models with crossed and nested diagonal random-effects structures with and without treatment effects.

\begin{tabular}{|c|c|c|c|c|c|c|c|}
\hline \multicolumn{2}{|r|}{ Model } & \multirow[t]{2}{*}{ AIC } & \multirow[t]{2}{*}{ BIC } & \multirow{2}{*}{$\begin{array}{l}\text { Log-like } \\
\text { lihood }\end{array}$} & \multirow[t]{2}{*}{ Test } & \multirow{2}{*}{$\begin{array}{l}\text { Likeli. } \\
\text { Ratio }\end{array}$} & \multirow[t]{2}{*}{ P-value } \\
\hline No. & Structures & & & & & & \\
\hline 1 & $\begin{array}{l}\text { Without trt effect } \\
\text { \& Crossed }\end{array}$ & -21095 & -20996 & 10562 & 1 vs 4 & 395.67 & $<.0001$ \\
\hline 2 & $\begin{array}{l}\text { Without trt effect } \\
\text { \& Nested }\end{array}$ & -20689 & -20544 & 10366 & 2 vs 4 & 787.73 & $<.0001$ \\
\hline 3 & $\begin{array}{l}\text { With trt effect \& } \\
\text { Crossed }\end{array}$ & -21322 & -20900 & 10725 & 3 vs 4 & 70.912 & $<.0001$ \\
\hline 4 & $\begin{array}{l}\text { With trt effect \& } \\
\text { Nested }\end{array}$ & -21379 & -20911 & 10760 & -- & -- & -- \\
\hline
\end{tabular}


Fig 2 Multiple plots of observed (black open circle) and predicted (grey solid line) Tb from mixed additive bilogistic model with treatments and diagonal nested random-effect structure. Missing values were excluded.

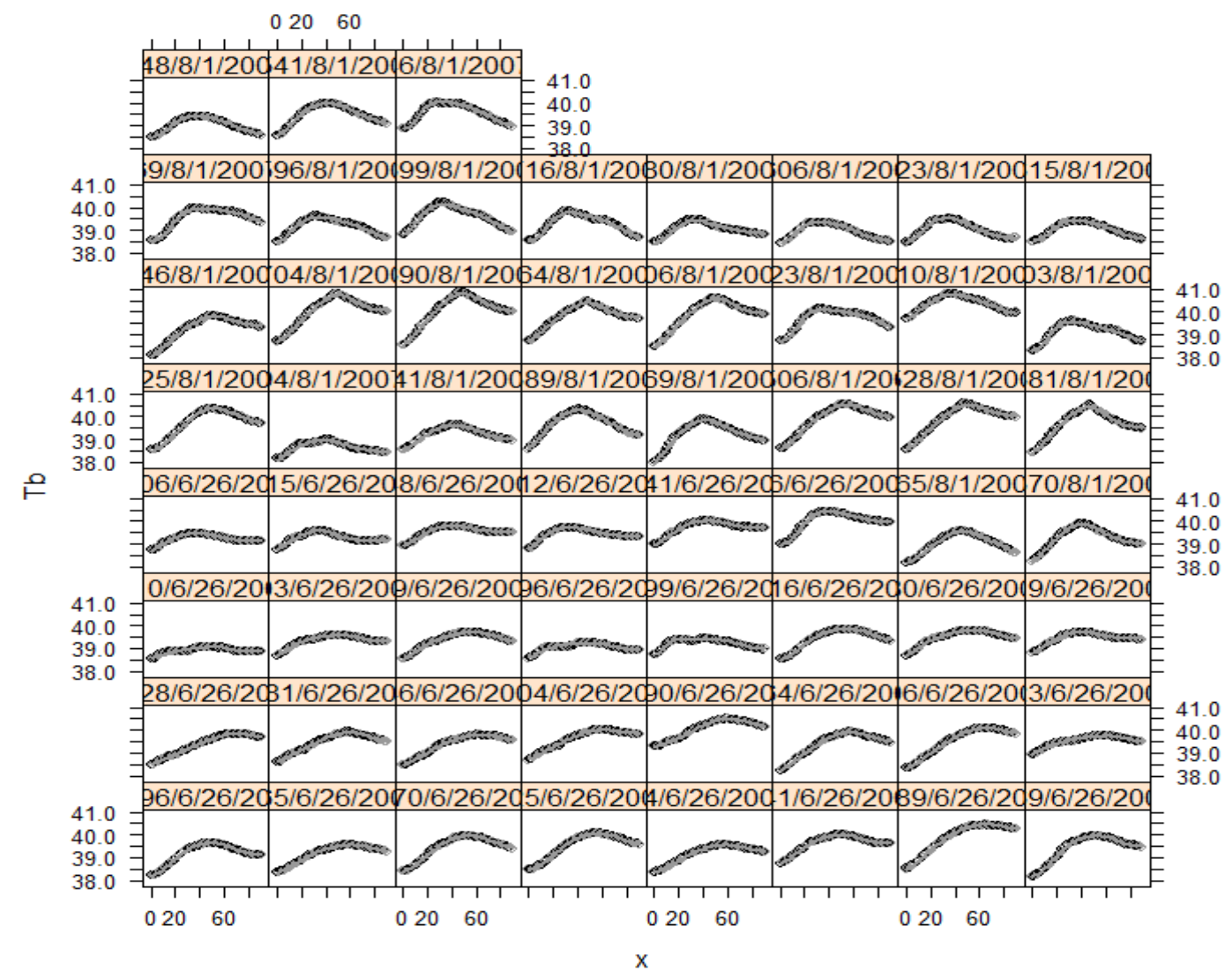

\subsection{Examination of Random Effects}

The full diagonal random-effects structure specified in (7) was summarized in Table 4. Estimated standard deviations for seven parameters at both heifer and day within heifer levels are necessary (p-values from LRT test were significant $(<0.05)$ which showed that the effects cannot be removed from the random structure). The residual for model 4 was 0.02610 which was significantly smaller than the residual for model 3 (0.02725, not shown). The nested structure with the random-effects among heifer and day within heifer could account for the variation which is due to heifer conditions and environmental factors. The interactions between heifer and day within heifer existed in all parameters.

Table 3. Standard deviations of Random-effects and Residual for Nonlinear Mixed Models with Nested Random Structure

\begin{tabular}{|l|l|l|l|l|l|l|l|l|}
\hline \multirow{2}{*}{$\begin{array}{l}\text { Parameters } \\
\text { Levels }\end{array}$} & \multicolumn{7}{c|}{ Standard Deviations } & \multirow{2}{*}{ Residual } \\
\cline { 2 - 9 } & $\alpha$ & $\delta$ & $T b_{\text {init }}$ & $k_{1}$ & $\mathrm{k}_{2}$ & $\tau_{1}$ & $\tau_{2}$ & \multirow{2}{*}{0.02610} \\
\hline Heifer & 0.0044 & 0.0041 & 0.0025 & 0.1033 & 0.0001 & 0.1512 & 0.0680 & 0.026 \\
\hline Day in heifer & 0.0089 & 0.0064 & 0.0054 & 0.3412 & 0.2399 & 0.8450 & 0.3022 & \\
\hline
\end{tabular}




\subsection{Examination of Treatment Effects}

The mixed model with hierarchical random-effects was used to determine the significance of the treatment effects associated with each parameter. The results were summarized in Table 5. The p-values for interactions are all significant $(<5 \%)$ except the recovery inflection point, $\tau_{2}$, which is essentially the change point - time at which the rate changes from decreasing to increasing. It indicates that there is no significant pen and day interaction for $\tau_{2}$. In addition, none of the Pen or Day effects are significant. The estimate of the fixed effect recovery change point (the inflection point of recovery) is $72.35 \mathrm{~min}$.

Table 4 ANOVA for testing treatment effects for each parameter (denominator d.f.=5255).

\begin{tabular}{|c|c|c|c|c|}
\hline \multicolumn{2}{|c|}{ Factor } & Num DF & F-value & P-value \\
\hline \multirow[t]{4}{*}{$\alpha$} & Intercept & 1 & 4741237 & $<.0001$ \\
\hline & Pen & 3 & 10389 & $<.0001$ \\
\hline & Day & 1 & 56555 & $<.0001$ \\
\hline & Pen X Day & 3 & 151213 & $<.0001$ \\
\hline \multirow{4}{*}{$\delta$} & Intercept & 1 & 10518321 & $<.0001$ \\
\hline & Pen & 3 & 1455 & $<.0001$ \\
\hline & Day & 1 & 3439 & $<.0001$ \\
\hline & Pen X Day & 3 & 3359 & $<.0001$ \\
\hline \multirow{4}{*}{$T b_{\text {init }}$} & Intercept & 1 & 18656897 & $<.0001$ \\
\hline & Pen & 3 & 12 & $<.0001$ \\
\hline & Day & 1 & 40 & $<.0001$ \\
\hline & Pen X Day & 3 & 3 & 0.0151 \\
\hline \multirow{4}{*}{$k_{1}$} & Intercept & 1 & 1777 & $<.0001$ \\
\hline & Pen & 3 & 9 & $<.0001$ \\
\hline & Day & 1 & 7 & 0.0065 \\
\hline & Pen X Day & 3 & 6 & 0.0005 \\
\hline \multirow{4}{*}{$k_{2}$} & Intercept & 1 & 3293 & $<.0001$ \\
\hline & Pen & 3 & 17 & $<.0001$ \\
\hline & Day & 1 & 33 & $<.0001$ \\
\hline & Pen X Day & 3 & 14 & $<.0001$ \\
\hline \multirow{4}{*}{$\tau_{1}$} & Intercept & 1 & 563 & $<.0001$ \\
\hline & Pen & 3 & 6 & 0.0008 \\
\hline & Day & 1 & 9 & 0.0036 \\
\hline & Pen X Day & 3 & 3 & 0.0476 \\
\hline \multirow{4}{*}{$\tau_{2}$} & Intercept & 1 & 9725 & $<.0001$ \\
\hline & Pen & 3 & 2 & 0.0960 \\
\hline & Day & 1 & 0 & 0.6358 \\
\hline & Pen X Day & 3 & 2 & 0.0644 \\
\hline
\end{tabular}

For all other parameters, the interactions between days and pens were examined. To help understand the significant day and pen interactions on the six parameters, $\alpha, \delta$, 
$T b_{\text {init }}, k_{1}, k_{2}$, and $\tau_{1}$, plots for the treatment means were examined, Fig 3. For each parameter, the day means were given by pen. The pen with the shortest distance was given first. If there was no interaction, the day line segments in the plot would be parallel. It is clear that there was an interaction between pens and days for each parameter in Fig 3.

Fig 3. Interaction plots of parameters for cool day (dashed) and hot day (solid). Pen closest to work station is given first. The overall mean (dotted) for each parameter is given.
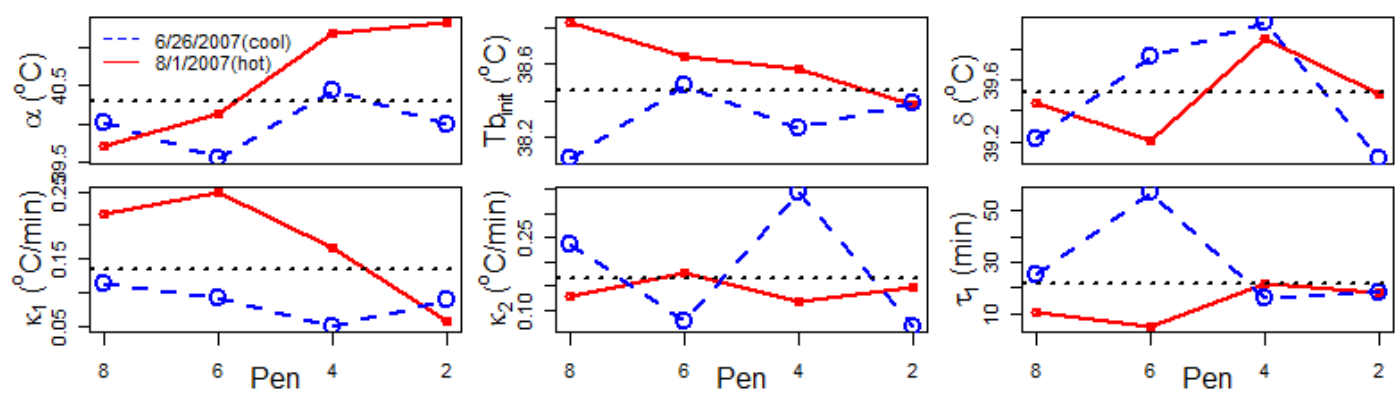

The upper asymptotic body temperature, $\alpha$, indicates the highest body temperature an animal is expected to achieve in the absence of a recovery process. The upper asymptote was higher for the hot day than it was for the cool day except for the pen 8 which was closest to the work station. An increasing trend was shown for the hot day as the distance of the pen from the work station increased. Overall, heifers moved farthest in a hot environment had a higher Tb asymptote.

The initial body temperature, $T b_{\text {init }}$, was higher for the hot day than it was for the cool day except for the most distant pen 2. For that pen the two days had essentially the same initial body temperature. Pens 8 and 4 had significantly higher initial Tb which may suggest characteristics of pens or animals not necessarily distant from work station.

Heifers in pens 6 and 4 had higher baseline Tb for recovery, $\delta$, on the cooler day. The baseline $\mathrm{Tb}$ for recovery in pen 4 was significantly higher than others (p-value=0.0256). It was above the overall mean on both days. This suggests the presence of other factors related to that pen interfere with the heifers' recovery and is a topic for further investigation.

The interaction plot of the initial challenge rate constant, $k_{1}$, showed a decreasing trend for the hot day. A higher initial challenge rate constant indicates a more rapid increase in Tb. On a hot day, heifers moved a short distance had a higher challenge rate constant, which implies $\mathrm{Tb}$ increased more rapidly than for heifers moved a longer distance. This result suggests that when heifers were challenged by heat stress, they took longer to adjust to handling the farther they were moved.

The recovery rate constant, $k_{2}$, did not change noticeably for the hot day; for the cool day, the rate constant was significantly different from the rest in pen 4 . The recovery 
rate constant in pen 4 was significantly higher than the overall mean (p-value $=0.0001$ ). Pen 4 was previously a matter of concern when considering the recovery baseline Tb. Once again this suggests the presence of other factors related to Pen 4 that hamper the heifers' recovery and is a topic for further investigation.

There was a significant interaction for the challenge inflection point, $\tau_{1}$, which is essentially the change point - time at which the rate changes from increasing to decreasing. This parameter is usually associated with the challenge rate constant. On the cool day, $\tau_{1}$ was significantly higher for the closest pens (8 and 6) while their rate constants were lower. This implies that heifers may need more time to adjust to the stress of handling when they were moved a short distance during a cool working environment. The opposite is true for the hot day. The heifer's inflection point is lower while the initial rate constant is higher suggesting heifers need to response more rapidly to the moving event.

\section{CONCLUSION}

An additive bilogistic model can be used to describe the dynamics of Tb during moving and handling of heifers. The additive nonlinear bilogistic mixed model with seven parameters, upper asymptote body temperature, initial body temperature, baseline body temperature for recovery, challenge and recovery rate constants and challenge and recovery inflection points, described the overall moving event in which both challenge and recovery were included. Regardless of random structures, treatment effects were necessary in the mixed model. Comparison of random effects favored the grouped heifer/day (nested) random structure over the heifer-day event (crossed) structure. Day and pen are two important factors that influence a heifer's Tb during stages of thermal challenge and recovery. Day-pen interactions occur in all parameters except the recovery inflection point. Characteristics of heifers moved from pens closer to the work station tend to be similar and depend on the day. Heifers moved the farthest on a hot day have a higher upper asymptote $\mathrm{Tb}$ and more rapid change in the increasing $\mathrm{Tb}$ than on a cool day. Although interaction effects from factors pen and day were found in initial $\mathrm{Tb}$, baseline Tb for recovery, recovery rate constant, and challenge inflection point, they may be caused by the unusual values in pen 4 or 8 . This implies other sources of variation, such as an animal's behavior, weight, and health history are present. Consequently, more care is required when moving animals larger distances during hot summer days. Further study of distance and heifer characteristics during thermally challenging conditions provide insight into the dynamics of heat stress.

\section{SUMMARY}

Heat stress can be a serious problem for animals in hot conditions. Understanding when and how heat stress happens would help to provide better ways to improve an animal's well being. Body temperature can be used to describe an animals' thermo-regulatory response to the environment. One way to model the process of handling and moving animals during thermal challenge and recovery in summer is to assume the processes are additive and utilize an additive bilogistic model. The model has seven parameters, initial body temperature, heat challenge rate constant, upper 
asymptote for body temperature, challenge inflection point, baseline for recovery, recovery rate constant, and recovery inflection point.

In this study, mixed additive bilogistic models with and without treatment effects using a nested random structure or a crossed random structure were compared. The mixed additive bilogistic model using a diagonal nested random structure with treatments was preferred. Treatment factors were significant for all parameters except the recovery inflection point. Significant pen and day interactions were found for the other six parameters. During a hot environment, heifers in pens farthest from the work station have a higher upper asymptote $\mathrm{Tb}$ and more rapid change in the increasing $\mathrm{Tb}$. Although interaction effects from factors pen and day were found in other four parameters (initial $\mathrm{Tb}$, baseline $\mathrm{Tb}$ for recovery, recovery rate constant, and challenge inflection point), they may come from some other sources of variation, such as an animal's behavior, weight, and health history, in some pens.

The mixed additive bilogistic model with nested random effect structure is helpful for predicting the change in heifer Tb during moving events. Moving cattle during a hot day or for a relatively larger distance in the summer produces heat stress and may produce economic loss. Besides day and pen, a heifer's condition, such as weight and health, needs to be considered when developing management strategies. However, moving distances and environment are two of the most important factors that need to be considered when moving animals.

\section{REFERENCES}

Aitha, N., A.M. Parkhurst, C.N. Lee, P.E. Hillman. 2008. Nonlinear mixed models to evaluate effects of environment conditions, hair coat, and anchor length on body temperature during afternoon milking of holstein cows in hawaii. Proc. of $20^{\text {th }}$ Annual Kansas State University Conference on Applied Statistics in Agriculture.206-217. Kansas State Univ. Manhattan, Kansas.

Brown-Brandl, T.M., R.A. Eigenberg, J.A. Nienaber. 2009. Water spray cooling during handling of feedlot cattle. International Journal of Biometeorology. Published online: http://www.springerlink.com/content/nm9n417261217624/

Davis, M.S., T.L. Mader, S.M. Holt, A.M. Parkhurst. 2003. Strategies to reduce feedlot cattle heat stress: effects on tympanic temperature. J Anim Sci 81:649-661

Kerek, M., A.M. Parkhurst, T.L. Mader. 2003. Using the bi-logistic model to estimate body temperature in feedlot cattle. Proc. of $15^{\text {th }}$ Annual Kansas State University Conference on Applied Statistics in Agriculture. 206-216. Kansas State Univ. Manhattan, Kansas.

Li, X., A.M. Parkhurst, T.L. Mader. 2009. Comparing experimental designs for A bi-logistic model used to estimate heat stress when moving feedlot cattle. Proc. of $21^{\text {st }}$ Annual Kansas State University Conference on Applied Statistics in Agriculture. 182-197. Kansas State Univ. Manhattan, Kansas.

Parkhurst, A.M. and T.L. Mader. 2000. Using nonlinear growth curves to estimate 
heat stress in processing feedlot cattle. Proc. of $12^{\text {th }}$ Annual Kansas State University Conference on Applied Statistics in Agriculture. Manhattan, Kansas. Pinheiro, J.C. and D.M. Bates. 2000. Mixed-effects models in S and S-PLUS. Springer. New York

Zhou, M., A.M. Parkhurst, R.A. Eigenberg, J. Nienaber, G.L. Hahn. 2006. Evaluating nonlinear crossed random effects models for comparing temperature of eating pigs under different thermal environments. Proc. of $18^{\text {th }}$ Annual Kansas State University Conference on Applied Statistics in Agriculture. 180-200. Kansas State Univ. Manhattan, Kansas. 